\title{
R. L. Wallace, T. W. Snell, C. Ricci, and T. Nogrady: Rotifera: Volume 1-Biology, Ecology and Systematics (2nd edn)
}

\author{
Backhuys Publishers, 2006, 299 pp, Paperback, €76.00, ISBN 90-5782-178-8
}

\author{
Thomas A. Davidson
}

Received: 29 June 2011/Accepted: 30 June 2011/Published online: 27 August 2011

(C) Springer Science+Business Media B.V. 2011

The fact that this review appears now, for a book published in 2006, in no way detracts from its relevance. This book provides an excellent background to Rotifer biology for both the specialist and relative newcomers to the world of rotifers, and will remain relevant for many years to come. It provides a revised and expanded update on the original edition published in 1993 by Nogrady, Wallace \& Snell. It consists of a number of sections providing a very thorough examination of rotifer biology and ecology, as well as detailed information on methodologies that can be used in rotifer studies.

The book starts with an introduction and general background as to why rotifers have fascinated microscopists over the years, while detailing some of their distinguishing features. The book is then divided into nine further chapters. Chapter 2: 'Techniques of collection, culture and preparation' provides an extremely useful start point for beginners in rotifer research, but it will also be invaluable for more experienced researchers. In particular, the table summarising the methods of collection and their limitations provides an excellent summary of the appropriate methods, which might be considered

T. A. Davidson $(\bowtie)$

Department of Bioscience, University of Aarhus, Aarhus, Denmark

e-mail: thd@dmu.dk before embarking on a piece of research. Chapter 3: 'Morphology and Internal Organization', provides a good general introduction to rotifer anatomy, muscular system, with details on trophi. The following chapter 'Physiology' outlines details on rotifer reproduction, locomotion, response to water quality change and metabolism. This leads into two more detailed chapters, 5: 'Population Ecology' and 6: 'Community Ecology', which form the core of the book and they provide a very complete review on the abiotic and biotic factors that are important in determining the population structure of rotifer assemblages, morphological variations, biogeography and dispersal. The community ecology section nicely summarises studies from a wide range of habitats. Furthermore, this chapter examines biological interactions, competition, predation and parasitism and knowledge of their role in food webs. Chapter 7 deals with the controversial subject 'Evolution of Rotifers', which even with the aid of modern molecular techniques is still a matter of debate. This leads neatly to chapters 8: 'Systematics, Taxonomy and Classification' and 9: 'Identification', which include a very useful guide to sources of information on rotifer identification available on the Internet. Chapter 10: 'A Brief History of Rotifer Research' then provides a nice summary of the people who have most shaped rotifer research. In conclusion, the book provides a very good grounding in all things rotifer-based and would be an invaluable resource not only to beginners in the topic, but also to more experienced researchers. 